Viewpoint

\section{The Paradox of Precision Medicine}

\author{
Roberta Diaz Brinton ${ }^{1, *}$, Lisa Mosconi ${ }^{2}$, Carolee Lee ${ }^{3}$, George Vradenburg 4 \\ 1 Center for Innovation in Brain Science; University of Arizona, 1230 N. Cherry \\ Ave., P.O. Box 210242, Tucson, AZ 85721, USA \\ 2 Department of Neurology, Weill Cornell Medicine, 420 East 70th St., New York, \\ NY 10021, USA \\ 3 Women's Health Access Matters, 19 East Elm St, Greenwich, CT 06830, USA \\ 4 UsAgainstAlzheimer's, P.O. Box 34565, Washington, D.C. 20043, USA \\ * Correspondence: Roberta Diaz Brinton, Email: rbrinton@arizona.edu.
}

Alzheimer's disease is an insidious ailment. It is the only top-10 deadly disease in the United States without an effective treatment or cure and one of the most financially burdensome diseases on American families and the broader healthcare system. There are unfortunately many "on-ramps" to Alzheimer's disease. Many ways individuals are susceptible to developing the disease with some of these starting as early as 10 to 20 years before symptoms present themselves [1].

The problem, of course, is that there are no "off-ramps", at least not yet. Scientists, clinicians, and researchers are increasingly investigating the Alzheimer's "interstate system"-where the on-ramps are, when they're open, and how likely someone is to take them based on a variety of physiological and behavioral factors.

Belying this approach is a tacit acknowledgment that individuals are different. This is not a radical idea, yet the field of scientific research has struggled to acknowledge this basic fact, especially when it comes to differences in chromosomal sex-based physiology. That can be hard to believe, considering what we know about how the pathologies of so many diseases manifest themselves differently in men versus women.

For example, it is a point of recurring surprise and consternation to those unfamiliar with the field of medical research that, until 1993, most testing done in clinical trials was conducted exclusively on men. And it wasn't until as recently as 2016 that the National Institutes of Health (NIH) mandated that female mice also be used in animal research funded by the U.S. government. Thankfully, remedies to these past blind spots are emerging in clinical trials and other research across a variety of therapeutic areas and disease states.

Alzheimer's presents a highly relevant example of why this matters. Despite the fact that nearly two-thirds of the 5.8 million individuals living with Alzheimer's disease in the United States are women, and that women are twice as likely as men to be diagnosed with the disease (and not simply because they live longer on average), animal studies on neurodegenerative of Creative Commons Attribution 4.0 International License. 
diseases that use only males outnumber those that include females at a rate of 5.5 to 1 [2].

Simply put, from a scientific perspective, outcomes of single-sex analyses are relevant to that sex. From a translational perspective, the clinical relevance is substantially narrowed.

In fact, when it comes to Alzheimer's research, a growing focus on sexand gender-based differences is revealing substantive, innovative breakthroughs in how we understand Alzheimer's disease pathology and its separate effects on the female and male brains. Closing the gap on Alzheimer's research in the female brain and physiology will accelerate interventions and treatments for more than 60 percent of people living with the disease [3]. Further, outcomes of research of the female Alzheimer's brain will enable a comparative analysis of differences and commonalities of the disease that would inform a precision medicine therapeutic approach.

In Alzheimer's disease pathology, chromosomal sex as a highly relevant variable is evident in the identification of Alzheimer's on-ramps. One such on-ramp is decline in the brain's energy system. Our research has shown in both basic and clinical research that mid-life endocrine aging in females (perimenopause to menopause) is associated with decline in ability of the brain to use glucose as its primary fuel, critical to generating ATP to drive the energy demands of the brain [4]. This transition in both endocrine function and brain glucose metabolism is unique to females and does not appear in the male brain at this age [4].

Another driver of Alzheimer's disease in women during this midlife aging transition is elevated inflammation and immune system responses. Functional shifts in both the metabolic and immune systems of the female brain may be prevented by promising, but not proven, estrogen therapy delivered during the early stages of the endocrine aging phase [5]. While potentially effective for females, estrogen therapy is not appropriate for males [6]. In contrast, androgen therapy in males might be an appropriate therapy should men exhibit comparable symptoms [7], but certain sideeffect profiles for both estrogen and androgen therapy may make these approaches prohibitive for some patients.

Differences in endocrine therapy for women and men are well accepted at this point [8], but they serve as an illustrative example of sexbased therapy which could be instructive when considering other therapeutic interventions to prevent and treat Alzheimer's. The same is true for differences which may exist between the sexes within what is called the "brain-gut axis" [9].

Going forward, it is critical to investigate sex-differences in the connection between and trajectories of aging and risk of Alzheimer's. While having common pathological hallmarks, it is likely that Alzheimer's will have different "on-ramps" which could require different "off-ramp" therapies. Alzheimer's research that embraces both the differences and 
commonalities across sexes will advance and accelerate prevention and treatment for this devastating disease.

The future of medicine is in tailored treatments that will address individuals and not a mathematical average in a spreadsheet. For Alzheimer's, that means precision in determining etiology and effective treatments.

It's time to ramp-up efforts to embrace a fundamental pillar for preventing and curing Alzheimer's disease: precision medicine starts with sex-based differences. The paradox of precision medicine is that in deeply investigating the physiology and impact of a disease on a single sex, we get ever closer to understanding paths forward in treatment and intervention that work for both men and women.

Inquiries into the specific physiology of the female brain could one day yield promising results that could be used to treat both women and men for Alzheimer's disease. Of course, as women remain two-thirds of all Americans living with Alzheimer's disease, a therapy that is even effective primarily in women would make immense strides in reducing the medical, financial, and emotional toll Alzheimer's exacts on American families.

\section{CONFLICTS OF INTEREST}

$\mathrm{RDB}, \mathrm{LM}$ and CL declare that they have no conflicts of interest. GV declares he holds financial interests in brainscope and a very nominal interest in chase allergan. Interests in united neuroscience and brain trust accelerator II fund are owned as part of family trusts.

\section{ACKNOWLEDGMENTS}

The authors acknowledge funding from National Institutes of Health, 5R01AG057931-03 (RDB and LM) and P01AG026572 (RDB).

\section{REFERENCES}

1. Brain Changes Detected 20 Years before Alzheimer's Symptoms. Available from: https://www.alzinfo.org/articles/brain-detected-20-years-alzheimerssymptoms. Accessed 2020 Oct 22.

2. Zucker I, Beery AK. Males still dominate animal studies. Nature. 2010;465(7299):690. doi: 10.1038/465690a

3. Andrew MK, Tierney MC. The puzzle of sex, gender and Alzheimer's disease: Why are women more often affected than men? Womens Health (Lond). 2018;14:1745506518817995.

4. Wang Y, Shang Y, Mishra A, Bacon E, Yin F, Brinton R. Midlife Chronological and Endocrinological Transitions in Brain Metabolism: System Biology Basis for Increased Alzheimer's Risk in Female Brain. Sci Rep. 2020;10:8528.

5. Wahjoepramono EJ, Asih PR, Aniwiyanti V, Taddei K, Dhaliwal SS, Fuller SJ, et al. The Effects of Testosterone Supplementation on Cognitive Functioning in Older Men. CNS Neurol Disord Drug Targets. 2016;15(3):337-43. 
6. Janicki SC, Schupf N. Hormonal Influences on Cognition and Risk for Alzheimer Disease. Curr Neurol Neurosci Rep. 2010 Sep;10(5):359-66.

7. Testosterone may improve mental function. Available from: https://www.health.harvard.edu/press releases/testosterone-and-memory. Accessed 2020 Oct 22.

8. Gillies GE, McArthur S. Estrogen Actions in the Brain and the Basis for Differential Action in Men and Women: A Case for Sex-Specific Medicines. Pharmacol Rev. 2010 Jun;62(2):155-98.

9. Jašarević E, Morrison KE, Bale TL. Sex differences in the gut microbiomebrain axis across the lifespan. Philos Trans R Soc Lond B Biol Sci. 2016 Feb 19;371(1688):20150122.

How to cite this article:

Brinton BD, Mosconi L, Lee C, Vradenburg G. The Paradox of Precision Medicine. Adv Geriatr Med Res. 2020;2(4):e200026. https://doi.org/10.20900/agmr20200026 